\title{
PAPER Uplink Blocking Probabilities in Priority-Based Cellular CDMA Networks with Finite Source Population
}

\author{
Vassilios G. VASSILAKIS $^{\dagger \mathrm{a})}$, Ioannis D. MOSCHOLIOS ${ }^{\dagger \dagger \mathrm{b})}$, Nonmembers, , \\ and Michael D. LOGOTHETIS ${ }^{\dagger \dagger c)}$, Member
}

\begin{abstract}
SUMMARY Fast proliferation of mobile Internet and high-demand mobile applications necessitates the introduction of different priority classes in next-generation cellular networks. This is especially crucial for efficient use of radio resources in the heterogeneous and virtualized network environments. Despite the fact that many analytical tools have been proposed for capacity and radio resource modelling in cellular networks, only a few of them explicitly incorporate priorities among services. We propose a novel analytical model to analyse the performance of a prioritybased cellular CDMA system with finite source population. When the cell load is above a certain level, low-priority calls may be blocked to preserve the quality of service of high-priority calls. The proposed model leads to an efficient closed-form solution that enables fast and very accurate calculation of resource occupancy of the CDMA system and call blocking probabilities, for different services and many priority classes. To achieve them, the system is modelled as a continuous-time Markov chain. We evaluate the accuracy of the proposed analytical model by means of computer simulations and find that the introduced approximation errors are negligible. key words: CDMA, network capacity, priority classes, Markov chain
\end{abstract}

\section{Introduction}

Due to the heterogeneous nature of next-generation cellular systems and proliferation of high-demand mobile applications, the incorporation of enhanced radio resource management techniques is an important and, at the same time, a very challenging task [1]. Hence, the development of efficient modelling tools to facilitate the design, analysis and evaluation of such systems is essential. This is especially true under the presence of user-generated multirate traffic.

Contrary to traditional cellular networks, where each cell is controlled by a Base Station (BS), modern network designs introduce a many-to-many relationship among BSs and cells. This was fuelled by the advances in small-cell design and appearance of schemes such as Coordinated Multi-Point (CoMP) [2]. Furthermore, the incorporation of Software-Defined Networking (SDN) and Network Function Virtualization (NFV) concepts in cellular networks, facilitates the cooperation of BSs without relying on the ex-

\footnotetext{
Manuscript received August 30, 2015.

Manuscript revised January 27, 2016.

${ }^{\dagger}$ V. G. Vassilakis is with the Computer Laboratory, University of Cambridge, Cambridge, United Kingdom

${ }^{\dagger}$ I. D. Moscholios is with the Dept. of Informatics \& Telecommunications, University of Peloponnese, Tripolis, Greece

${ }^{\dagger}$ M. D. Logothetis is with the Dept. of Electrical \& Computer Engineering, University of Patras, Patras, Greece

a)E-mail: vv274@cl.cam.ac.uk

b)E-mail: idm@uop.gr

c) E-mail: mlogo@upatras.gr

DOI: 10.1587/transcom.E0.B.1
}

pensive mobile core infrastructure [3]. However, in order for these technological advances to be practical in the Radio Access Network (RAN), fast and efficient algorithms should be implemented at the BS-level [4].

Among other candidates, Code Division Multiple Access (CDMA) techniques are expected to be part of the nextgeneration cellular networks, due to their efficient spectrum utilization, improved signal quality and good security features [5], [6]. Due to non-orthogonality of CDMA codes, when a new Mobile User (MU) is accepted in the cell, it causes interference to other MUs in the same and in the neighbouring cells. Therefore, to protect the quality of service ( $\mathrm{QoS})$ of ongoing connections, a BS performs Call Admission Control (CAC) on the incoming calls. This means that some calls may be blocked, or given a lower transmission rate compared to what they requested.

In this paper, we develop a mathematical model to facilitate appropriate allocation of radio resources in the uplink of a CDMA cell. The model is based on a ContinuousTime Markov Chain (CTMC), which is used to describe the process of call arrivals and departures. We consider the so-called quasi-random call arrival process, which is more realistic for cellular networks (especially when considering dense small cell deployments) than the widely used Poisson process [7]. We also consider different priority classes, which receive different treatment during the CAC: In the event of traffic congestion in a cell, low priority calls may be blocked, depending on (predefined) thresholds. Since our goal is to develop a fast and highly efficient resource allocation algorithm for next-generation SDN-based BSs, we base our solution on the well-known Kaufman-Roberts (K-R) algorithm [8], [9], which possesses the desired properties and has been used in the past for wired (e.g., [10], [11]), wireless (e.g., [12], [13]) and optical (e.g., [14], [15]) networks. We extend the K-R algorithm to incorporate the peculiarities of priority-based CDMA networks and quasi-random arrivals.

In our previous model, proposed in [16], we also considered the peculiarities of CDMA systems, when modelling of the priority traffic classes was based on an extension of $\mathrm{K}-\mathrm{R}$ recursion. However, the calculation of call blocking probabilities (CBP) was performed assuming Poisson call arrival process and two priority classes, only. We now extend [16] by incorporating: (i) the quasi-random call arrival process, which basically means that a finite (arbitrary) number of traffic sources can be supported and (ii) an arbitrary finite number of priority classes. 
This paper is structured as follows: In Section 2, we briefly discuss the related work. In Section 3, we describe the considered model for cellular CDMA. In Section 4, we model the system as a CTMC, and derive efficient formulae for the calculation of system state probabilities and CBP. In Section 5, we evaluate the accuracy of the proposed algorithm by means of simulation. We conclude in Section 6 .

\section{Related Work}

We briefly review the related work, other than the already mentioned [16], by presenting the most important analytical models for CDMA systems, focusing on those that result in recurrent and efficient formulae. We also present a few works dealing with priority-based CAC in cellular networks.

\subsection{CDMA modelling}

A number of important teletraffic models have been proposed for the calculation of CBP in cellular CDMA networks. In [17], the K-R recursion is extended to incorporate the peculiarities of Wideband CDMA (W-CDMA) systems. The model results in an efficient formula for the calculation of CBP assuming Poisson call arrival process in the uplink direction and fixed cell resource requirements. This work was extended in [18] and [19] by incorporating into the model the quasi-random call arrival process. The model of [19] has been further extended in [20] to incorporate the batched Poisson input traffic, in CDMA networks. In [21], an efficient model for traffic overflow in cellular networks is proposed. The proposed approach simplifies the determination of the parameters of overflow traffic, such as its average value and variance. The model proposed in [22] uses a twostate Markov chain for the analysis of the radio interface in a W-CDMA network carrying a mixture of multi-rate traffic streams. The model enables quick determination of the average time that the system spends in a particular state; its accuracy is confirmed via simulations.

\subsection{Priority-based CAC}

There have been a few notable works that study the prioritybased CAC in cellular systems. A distributed priority-based CAC for cellular networks is proposed in [23]. The derived algorithm shows good adaptation properties in highly dynamic scenarios and under the presence of prioritized user mobility. High and low priority users are distinguished based on their target signal-to-noise ratio (SNR). In [24], a methodology for modelling and optimizing the radio access in W-CDMA is proposed. This approach takes into consideration different traffic priorities and results in a simple computational algorithm to determine CBP. The main difference between our present work and [24] is that in [24], the authors consider a non-full-availability group to model the W-CDMA interface. On the contrary, we consider the complete sharing policy, which means that all MUs compete for all available resources [25]. Another key difference is that in
[24], the authors assume as input traffic, Poisson traffic instead of quasi-random traffic. In [24], the authors also consider as input traffic, traffic at packet level, by exploiting the notion of equivalent bandwidth (variable bit rates of packet streams are replaced by constant bit rates, called equivalent bandwidth). However, even in this case, the arrival process is considered Poisson.

\section{System Model for Cellular CDMA}

In this section, we describe our considered model for a cellular CDMA system, and introduce the necessary assumptions and notations. Let us consider a CDMA system that consists of a reference cell surrounded by neighbouring cells. The system supports $K$ independent services and $P$ priority classes. Calls of a service may have different priorities.

Different services are distinguished according to their required data transmission rate. The rate of a service $k$ $(k=1, \ldots, K)$ call is denoted by $R_{k}$. The number of traffic sources of service $k$ is denoted by $S_{k}$. As we will see later, the classification of a call into one of the priority classes will influence the acceptance/blocking of the call during the CAC. A call of service $k$ and priority $p(p=1, \ldots, P)$ is referred to as a type $(k, p)$ call. The number of sources of service $k$ with priority $p$ is denoted by $S_{k, p}$. Hence, $S_{k}=\sum_{p=1}^{P} S_{k, p}$.

We model the uplink of a CDMA system, i.e., calls from MUs to BS. We adopt the following assumptions about the traffic generated by MUs. The inter-arrival time of class $p$ calls from an idle source of service $k$ is denoted by $\lambda_{k, p}$ and is assumed to be exponentially distributed. This forms the so-called quasi-random call arrival process [25], [26]. The requested service times are generally distributed. The mean service rate for a $(k, p)$ call is denoted by $\mu_{k, p}$. Hence, the offered traffic load per idle source in Erlangs (erl) for service $k$ and priority class $p$ is calculated as follows:

$$
a_{k, p}=\frac{\lambda_{k, p}}{\mu_{k, p}}
$$

We assume perfect power control at the BS of the CDMA cell [27]. This essentially means that the received signal power at the BS is the same from all MUs (of the same service) and does not depend on MU's location within the cell, or on the wireless propagation model. At the BS, the received power from a service $k$ call is denoted by $P_{k}$. Due to Multiple Access Interference (MAI), which is inherent in CDMA systems [28], the signals that are received at the BS from different MUs cause interference to each other. This is the case for MUs that reside in the same or in neighbouring cells. We also consider the thermal noise, which corresponds to the interference of an empty system. Therefore, to incorporate MAI in our model, we distinguish the following types of interference:

- the intra-cell interference, $I_{\text {intra }}$

- the inter-cell interference, $I_{\text {inter }}$

- the thermal noise, whose power is denoted as $P_{\text {noise }}$. 
Hence, the total interference, $I_{\text {total }}$, at the BS is calculated as:

$$
I_{\text {total }}=I_{\text {intra }}+I_{\text {inter }}+P_{\text {noise }}
$$

We also take into account the varying activity of MUs in terms of radio resources consumption. We assume that during the call duration there will be some active periods, when an MU transmits (and consumes cell radio resources) and passive periods, when an MU is either idle or receives (and does not consume any radio resources). We use the notion of activity factor $v_{k}\left(0<v_{k} \leq 1\right)$ for each service $k$ and model MU's behaviour as a Bernoulli random variable. The activity factor is defined as the ratio of the total duration of active periods over the whole call duration.

In CDMA systems, due to MAI, acceptance of a new call may degrade the perceived quality of in-service calls. Therefore, as mentioned in Section 1, CAC is usually performed for new calls. In typical CDMA systems, the CAC is performed by

- measuring the noise rise, $N R$, which is defined as the ratio of $I_{\text {total }}$ over $P_{\text {noise }}$

- evaluating $N R$ against the predefined CAC threshold, $N R_{\max }$

To model different priority classes, we associate each class $p$ with a CAC threshold $N R_{p, \text { max }}$. In general, $N R_{p, \text { max }}$ is a decreasing function with respect to $p$, i.e., calls of higher priority (smaller $p$ ) are expected to have a higher CAC threshold.

To represent the CDMA system resources, we adopt the notion of cell load, CL, defined in (3). This quantity is well suited for the modelling of cellular resources at the call level and has also been used in other works (e.g., [17], [20]):

$$
C L=\frac{I_{\text {total }}-P_{\text {noise }}}{I_{\text {total }}}
$$

where $0 \leq C L \leq 1$. The upper bound for the cell load is achieved when $P_{\text {noise }} \rightarrow 0$. However, in practice a typical upper bound is less than 1 and is, e.g., $C L_{\max }=0.8$ [19].

After the adoption of the cell load as the shared system resource, we define a suitable quantity for resource requirements of a call. We adopt the call's load factor, defined in (4) for service $k$, which depends on the transmission rate, $R_{k}$, the SNR, $S N R_{k}$, and the carrier's chip rate, $W$.

$$
L F_{k}=\frac{R_{k} S N R_{k}}{R_{k} S N R_{k}+W}
$$

The cell load can be further decomposed into the intracell load, $C L_{\text {intra }}$, caused by MUs of the same cell, and the inter-cell load, $C L_{\text {inter }}$, caused by MUs of the neighbouring cells. These are defined in (5) and (7), respectively.

$$
C L_{\text {intra }}=\sum_{k=1}^{K} \sum_{u=1}^{U_{k}} L F_{k} 1(u, k)
$$

where $U_{k}$ is the number of service $k$ MUs within the cell at a given time and $1(u, k)$ is the activity indicator function for user $u\left(u=1, \ldots, U_{k}\right)$ of service $k$, defined in (6), below.

$$
\begin{aligned}
1(u, k) & = \begin{cases}1, & \text { if user } u \text { of service } k \text { is active } \\
0, & \text { if user } u \text { of service } k \text { is passive }\end{cases} \\
C L_{\text {inter }} & =\left(1-C L_{\text {max }}\right) \frac{I_{\text {inter }}}{P_{\text {noise }}}
\end{aligned}
$$

\section{Uplink Blocking Probabilities}

\subsection{Local Blocking Probabilities}

In this subsection, we determine the so-called Local Blocking Probabilities (LBPs), which refer to call blocking in a particular system state. As already mentioned, the cell load, $C L$, can be decomposed into $C L_{\text {intra }}$ and $C L_{\text {inter }}$. Since the $\mathrm{BS}$ is aware of the number of in-service calls in its cell, $C L_{\text {intra }}$ can be determined from (5) taking into account the number of MUs of each service and their corresponding load factors. On the other hand, the accurate determination of $C L_{\text {inter }}$ may not always be possible. This is because generally a BS is not aware of the number of MUs at the neighbouring cells and their services. Although, there are proposed schemes that allow cooperation among BSs to exchange such type of information (e.g., [29]), we do not consider this assumption, since many operators avoid such schemes. As it has been shown in [16], [17], $C L_{\text {inter }}$ can be modelled as a log-normal random variable with mean $\mu=E\left[C L_{\text {inter }}\right]$ and variance $\sigma^{2}=V A R\left[C L_{\text {inter }}\right]$. The Cumulative Distribution Function $(\mathrm{CDF})$ of $C L_{\text {inter }}$ is given by:

$$
F(x)=\frac{1}{2}\left(1+\operatorname{erf}\left(\frac{\ln x-\mu}{\sigma \sqrt{2}}\right)\right)
$$

where erf() is the well-known error function.

Since the BS is explicitly aware of $C L_{\text {intra }}$, but not of $C L$ or $C L_{\text {inter }}$, we express the probability of a call being blocked, as a function of $C L_{\text {intra }}$. This is the LBP and is defined for a service $k$ and a priority class $p$ as follows:

$$
\begin{aligned}
& L B P_{k}^{p}\left(C L_{\text {intra }}\right)=\operatorname{Pr}\left[C L_{\text {intra }}+C L_{\text {inter }}\right. \\
& \left.+L F_{k}>C L_{p, \text { max }}\right]
\end{aligned}
$$

where $C L_{p, \max }$ is the CAC threshold for class $p$ calls.

By performing some manipulations with (8) and (9), we derive an analytical expression for LBPs:

$$
L B P_{k}^{p}\left(C L_{\text {intra }}\right)= \begin{cases}1-F(x), & \text { for } x \geq 0 \\ 1, & \text { for } x<0,\end{cases}
$$

where $x=C L_{p, \max }-C L_{\text {intra }}-L F_{k}$.

\subsection{State Probabilities}

Having defined in Section 3 the cell load as a shared system resource and the call's load factor as the resource requirement of a call, we describe the process of call arrivals and departures as a CTMC. To this end, we modify the K-R recursion for the calculation of state probabilities, in order to 


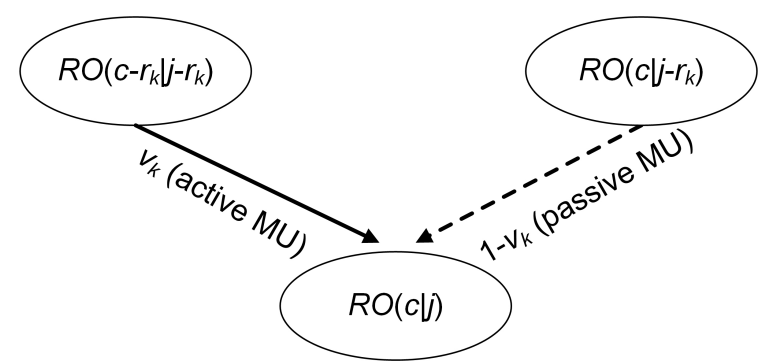

Fig. 1 Recursive calculation of the Resource Occupancy (general case).

derive a time- and space-efficient algorithm.

The necessary steps for the modification of K-R recursion are as follows. First of all, the CTMC modelling requires the discretization of $C L_{p, \max }$ and $L F_{k}$. This is performed with the use of the basic discretization unit, $g$ :

$$
C=\frac{C L_{p, \max }}{g}, r_{k}=\frac{L F_{k}}{g}
$$

where $C$ is the system capacity and $r_{k}$ is the resource requirement of a service $k$ call, in the corresponding K-R recursion [8].

Let us denote by $c$ the total number of occupied resources in the cell. This can be calculated by adding the load factors of all active MUs in the cell:

$$
c=\sum_{k=1}^{K} \sum_{u=1}^{U_{k}} r_{k} 1(u, k)
$$

By comparing (12) with (5), we can notice that if $C L_{\text {intra }}$ is known, then $c$ can be determined with the aid of the discretization unit, $g$, as follows:

$$
c=\frac{C L_{\text {intra }}}{g}
$$

Let us denote by $j$ the total number of resources that would be occupied in the cell if all MUs were active. In this case, we should take into account the "resource requirements" of passive MUs as well. The parameter $j$ can be calculated as follows:

$$
j=\sum_{k=1}^{K} \sum_{u=1}^{U_{k}} r_{k}=\sum_{k=1}^{K} U_{k} r_{k}
$$

Note that the only difference between (12) and (14) is that in the latter no activity indicator function is used. This means that $c$ captures the real resource consumption at a given moment, whereas $j$ reflects the "worst-case" scenario, where all passive MUs suddenly switch to active mode. From the above definitions of $c$ and $j$ it follows that $0 \leq c \leq j$. For a given $j$, if all MUs are passive, then $c=0$. Consequently, if all MUs are active, then $c=j$.

In the following, $j$ will be considered as the system (macro-)state while its maximum value is denoted by $j_{\max }$. Also, the situation in which $c$ resources are occupied in state $j$ will be referred to as system micro-state $(c, j)$. Note that

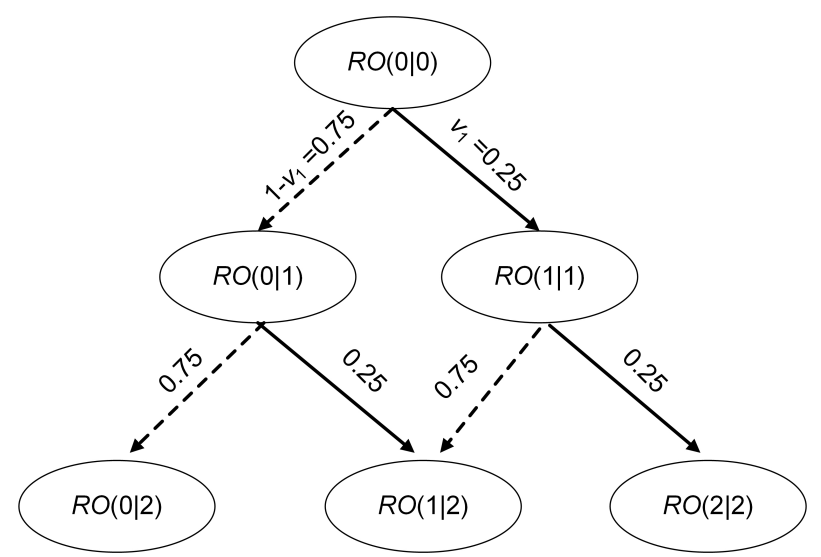

Fig. 2 Calculation of the Resource Occupancy (simple example).

the micro-states have been introduced merely to facilitate the explanation of (15), below, and should not be seen as the states of the CTMC, for which $j$ is used. Based on the previous discussion it is clear that the number of occupied resources in a state $j$ is not fixed, but may significantly vary depending on user activity. Hence, to capture this dependency, we introduce the Resource Occupancy (RO), $R O(c \mid j)$, which is defined as the conditional probability that $c$ resources are occupied in state $j$ :

$$
\begin{aligned}
& R O(c \mid j)=\sum_{k=1}^{K} R S_{k, p}(j)\left[v_{k} R O\left(c-r_{k} \mid j-r_{k}\right)\right. \\
& \left.+\left(1-v_{k}\right) R O\left(c \mid j-r_{k}\right)\right]
\end{aligned}
$$

for $j=1, \ldots, j_{\max }$ and $0 \leq c \leq j$, with the initial conditions $R O(0 \mid 0)=1$ and $R O(c \mid j)=0$ for $c>j$, where $v_{k}$ is the activity factor of service $k$ calls and $R S_{k, p}(j)$ is the Resource Share (RS) of service $k$ in a state $j$ (which is calculated in (20), below).

The basic concepts behind the recursive calculation of $R O(c \mid j)$ (i.e., (15)) are explained below with the aid of Figs. 1 and 2. Figure 1 represents the general case, whereas Fig. 2 illustrates the specific case of the tutorial example given below. Note, that these figures do not correspond to state transition diagram of the CTMC, since, as mentioned above, the micro-states $(c, j)$ do not correspond to actual states of the CTMC .

To understand the basic concept behind the recursive derivation of (15) let us consider a tutorial example. Assume that $K=1$ and $r_{1}=1$ and at a given time the system is in state $j=2$. Since $j=2$, two MUs are present in the cell (because $2 r_{1}=j$ ). Due to the constraint $0 \leq c \leq j$, we can have $c=0, c=1$, or $c=2$. Assume that $c=1$, which means that among the two MUs, one is passive and one is active. Let us now derive the following ROs: $R O(0 \mid 2)$, $R O(1 \mid 2)$, and $R O(2 \mid 2)$. To simplify the calculations, let us assume that only one priority class is supported (i.e., $P=1$ ) and the activity factor is $v_{1}=0.25$. We start from the initial micro-state $(0,0)$, where no MUs are present in the cell, with the initial condition $R O(0 \mid 0)=1$. This initial condition can be derived from the definition of the RO consid- 
ering the fact that when $j=0$, we have $c=0$ with probability 1 . Next, either an active or a passive MU will arrive in the cell. An active MU will arrive with a probability $\operatorname{Prob}$ [active arrival] $=\mathrm{v}_{1}=0.25$, whereas a passive MU will arrive with a probability $\operatorname{Prob}$ [passive arrival] = $1-\mathrm{v}_{1}=0.75$. Therefore, $R O(1 \mid 1)=v_{1} R O(0 \mid 0)=0.25$ and $R O(0 \mid 1)=\left(1-v_{1}\right) R O(0 \mid 0)=0.75$. Note that, since $K=1$ and $P=1$, all MUs are of the same type (i.e., same service and priority class). Therefore $R S_{k, p}(j)=1, \forall j$ and this parameter, although present in (15), is omitted from the calculations of this example.

Having determined $R O(0 \mid 1)$ and $R O(1 \mid 1)$, the derivation of the $\mathrm{RO}$ of micro-state $(1,2)$, denoted by $R O(1 \mid 2)$, can be performed recursively and in accordance with (15) and Fig. 2, as follows: We notice that there are two micro-states, namely $(0,1)$ and $(1,1)$, from which the transition to $(1,2)$ can occur upon the arrival of an MU. Using the language of probabilities, we get: $R O(1 \mid 2)=v_{1} R O(0 \mid 1)+\left(1-v_{1}\right) R O(1 \mid 1)$, which is a special case of (15). This concept has also been depicted in Fig. 1 in a more general context. Continuing the calculation, we get $R O(1 \mid 2)=0.1875+0.1875=0.375$. follows:

Similarly, the derivation of $R O(0 \mid 2)$ and $R O(2 \mid 2)$ is as

- $R O(0 \mid 2)=\left(1-v_{1}\right) R O(0 \mid 1)$, because the only way to reach the micro-state $(0,2)$ via an arriving MU is when the previous micro-state is $(0,1)$ and the arriving $\mathrm{MU}$ is passive. Hence, $R O(0 \mid 2)=0.75 * 0.75=0.5625$.

- $R O(2 \mid 2)=v_{1} R O(1 \mid 1)$, because the only way to reach the micro-state $(2,2)$ via an arriving MU is when the previous micro-state is $(1,1)$ and the arriving MU is active. Hence, $R O(2 \mid 2)=0.25 * 0.25=0.0625$.

Finally, we confirm that, as it was anticipated, $\sum_{c=0}^{c=j} R O(c \mid j)=$ $0.5625+0.375+0.0625=1$.

Due to MAI in CDMA systems, blocking of a call may occur at any state $j$. This is because the admission of calls in neighbouring cells may affect the capacity of the reference cell. Also, due to unpredicted activity, passive MUs may suddenly become active and thus quickly consume the available cell resources. Hence, we need to define the probability of call blocking at different system states. For this purpose, we introduce the State Blocking Factor (SBF). The SBF of service $k$ and priority class $p$ in state $j$, can be calculated by summing LBPs over $c$ and by multiplying with the corresponding ROs:

$$
S B F_{k}^{p}(j)=\sum_{c=0}^{j} L B P_{k}^{p}(c) R O(c \mid j)
$$

Below, we calculate the RS, that is used in (15). $R S_{k, p}(j)$ of service $k$ and priority class $p$ in state $j$ is defined as the ratio of resources occupied by a particular service $k$ and class $p$ over the total number of occupied resources in a given state $j$.

$$
R S_{k, p}(j)=\frac{E\left[U_{k, p}\right] r_{k}}{j}
$$

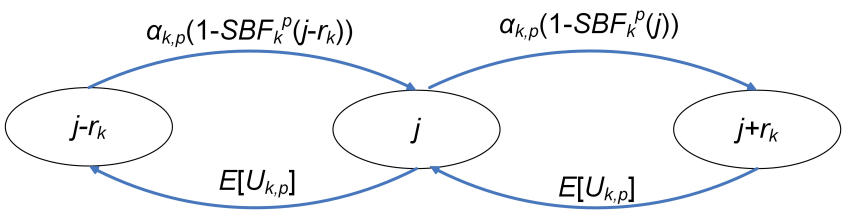

Fig.3 State transition diagram among adjacent system states.

where $E[x]$ is the expected value of a random variable $x$ and $U_{k, p}$ is the number of service $k$ and priority $p$ users with the condition $U_{k}=\sum_{p=1}^{P} U_{k, p}$. Note that, according to the above definition, $R S_{k, p}(j)$ is dimentionless.

We start with the assumption of local balance between adjacent system states, $j-r_{k}$ and $j$ :

$$
a_{k, p}\left(1-S B F_{k}^{p}\left(j-r_{k}\right)\right) q\left(j-r_{k}\right)=E\left[U_{k, p}\right] q(j)
$$

where $q(j)$ is the probability of state $j$. The corresponding state transition diagram is given in Fig. 3 .

Next, we multiply both sides of (18) by $r_{k}$ and divide by $j q(j)$, so that the right-hand side resembles that of (17):

$$
\frac{a_{k, p}\left(1-S B F_{k}^{p}\left(j-r_{k}\right)\right) r_{k} q\left(j-r_{k}\right)}{j q(j)}=\frac{E\left[U_{k, p}\right] r_{k}}{j}
$$

Hence, from (17) and (19) we get

$$
R S_{k, p}(j)=\frac{a_{k, p}\left(1-S B F_{k}^{p}\left(j-r_{k}\right)\right) r_{k} q\left(j-r_{k}\right)}{j q(j)}
$$

Having determined SBF and RS, we can derive the state probabilities, $q(j)$. We omit the detailed calculations and present the recursive formula for state probabilities:

$$
\begin{aligned}
& q(j)=\frac{1}{j} \sum_{k=1}^{K} \sum_{p=1}^{P}\left(S_{k, p}-E\left[U_{k, p}\right]+1\right) a_{k, p}(1- \\
& \left.S B F_{k}^{p}\left(j-r_{k}\right)\right) r_{k} q\left(j-r_{k}\right)
\end{aligned}
$$

for $j=1, \ldots, j_{\max }$ and $q(j)=0$ for $j<0$, with the condition $\sum_{j=0}^{j_{\max }} q(j)=1$.

\subsection{Call Blocking Probabilities}

The CBP $B_{k, p}$ of service $k$ and priority $p$ calls can be calculated by adding the probability of each state, $j$, multiplied by the corresponding SBFs:

$$
B_{k, p}=\sum_{j=0}^{j_{\max }} q(j) S B F_{k}^{p}(j)
$$

\section{Performance Evaluation}

We present numerical examples to evaluate the accuracy of the proposed analytical model. To this end, we compare the analytical versus simulation results in respect of CBP for different services and different priority classes. 


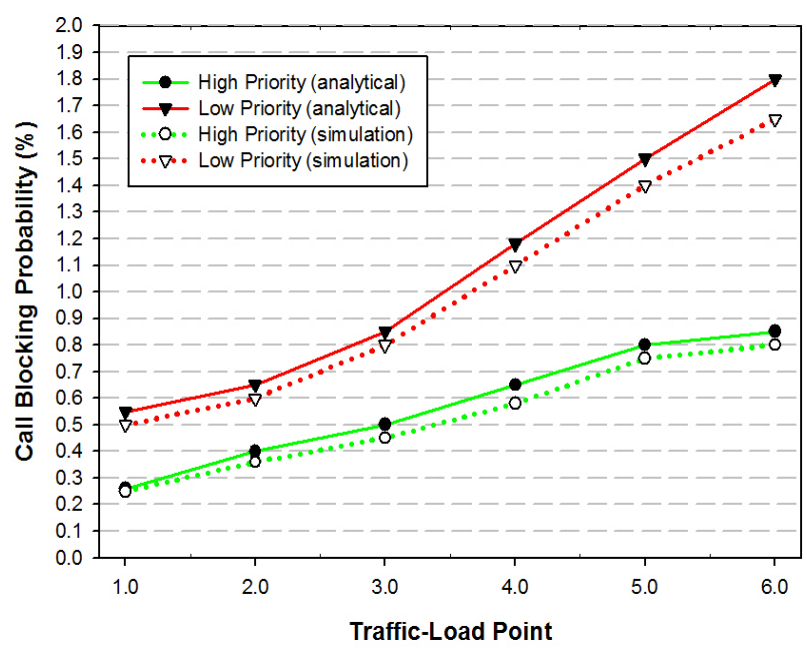

Fig. 4 Call blocking probabilities vs offered traffic for the 1st service.

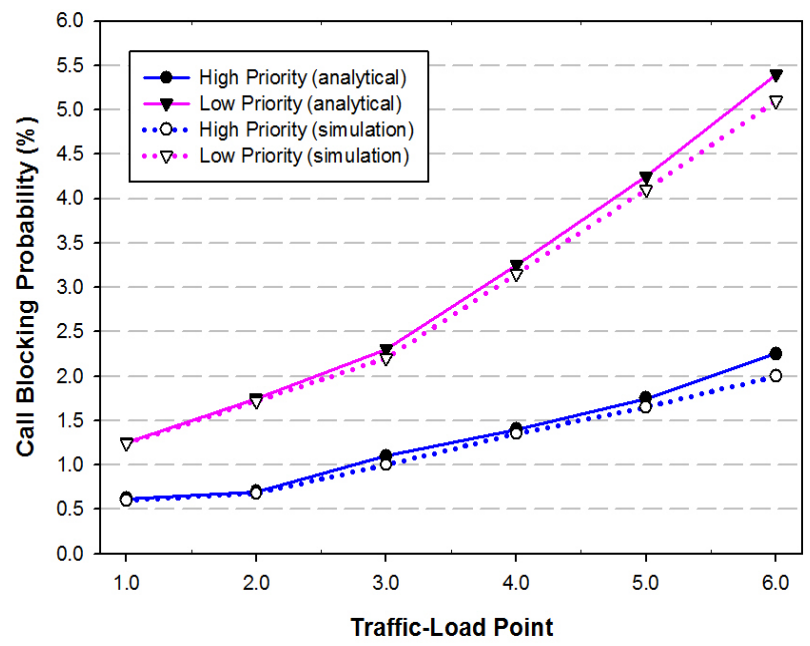

Fig. 5 Call blocking probabilities vs offered traffic for the 2nd service.

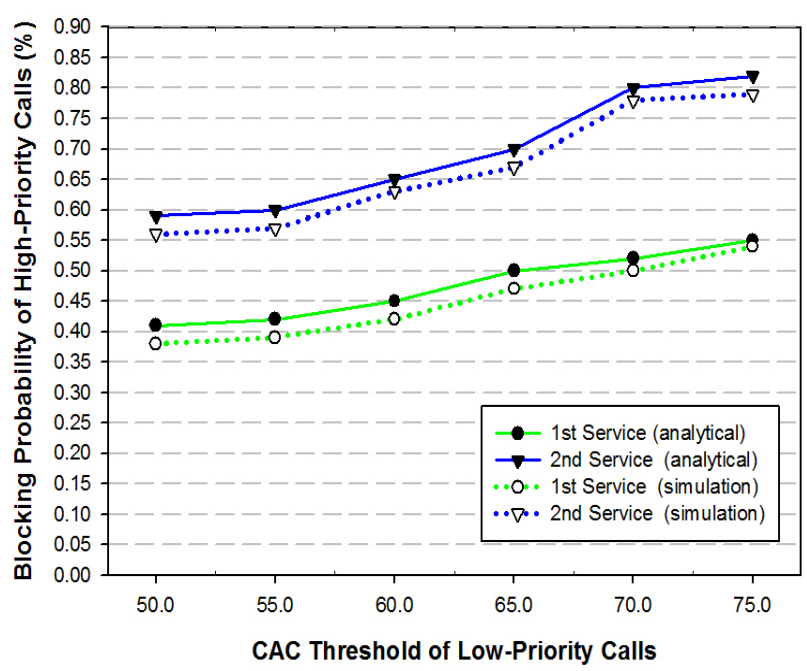

Fig. 6 Blocking probability of high priority calls vs CAC threshold of low priority calls (low traffic-load).

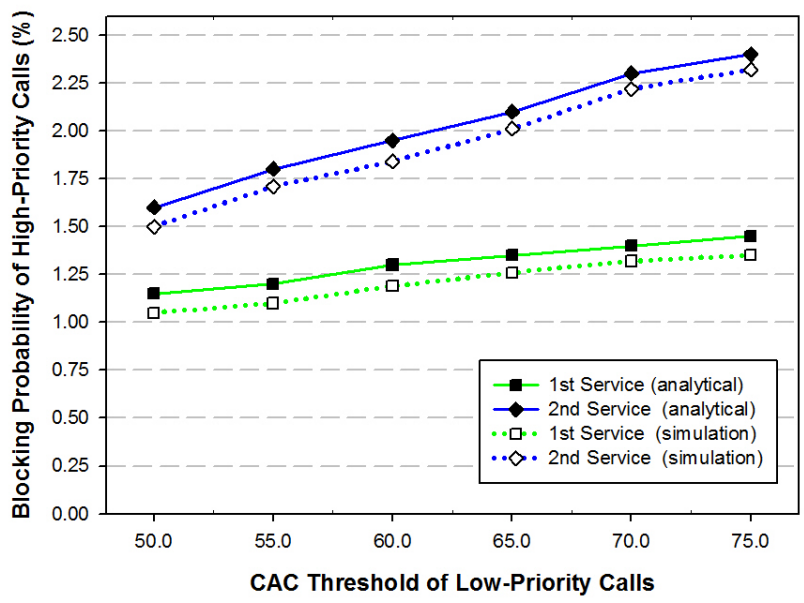

Fig. 7 Blocking probability of high priority calls vs CAC threshold of low priority calls (high traffic-load).

Table 1 Service Parameters

\begin{tabular}{|l|l|l|}
\hline & \multicolumn{1}{|c|}{$1^{\text {st }}$ Service } & \multicolumn{1}{|c|}{$2^{\text {nd }}$ Service } \\
\hline Data Rate & $R_{1}=144 \mathrm{Kbps}$ & $R_{2}=384 \mathrm{Kbps}$ \\
\hline High Priority Sources & $S_{1,1}=20$ & $S_{2,1}=10$ \\
\hline Low Priority Sources & $S_{1,2}=100$ & $S_{2,2}=50$ \\
\hline Activity Factor & $v_{1}=0.7$ & $v_{2}=1.0$ \\
\hline Signal-to-Noise Ratio & $S N R_{1}=3 \mathrm{~dB}$ & $S N R_{2}=4 \mathrm{~dB}$ \\
\hline
\end{tabular}

In Table 1, we present the necessary service parameters. Our analytical results are based on the formulae derived in Section 4. The state probabilities and CBP are calculated according to (21) and (22), respectively. Recall, that the calculation of state probabilities is based on approximations, due to incorporation of SBFs. Therefore, the comparison with simulation results intends to show that the impact of the approximation errors is negligible. The simulation of the cellular CDMA network is done using Simscript III [30]. 10 million calls were generated. The presented simulation results are mean values of 10 repetitions with $95 \%$ confidence interval. Since the resultant reliability ranges are very small, in the figures, we present only the mean values. The CAC has been implemented at the BS according to the description in Section 3. For each service, we record the number of blocked calls and calculate their ratio over the total number of arriving calls to determine the CBP. Note that we do not simulate neither the wireless propagation model nor the MU location in the cell. The reason is that, as was mentioned in Section 3 (see also [17]), at the BS of CDMA networks power control can be performed, in order for the received signal to be the same for all MUs (of a particular service) and independent on the MU location within the cell.

For the first service, we initially generate high-priority traffic of 0.02 erl and low-priority traffic of 0.1 erl (see also Table 2.). We then increase the high-priority traffic up to $0.12 \mathrm{erl}$ in steps of $0.02 \mathrm{erl}$, and the low-priority traffic up to $0.6 \mathrm{erl}$ in steps of $0.1 \mathrm{erl}$. For the second service, we initially generate high-priority traffic of 0.01 erl and low- 


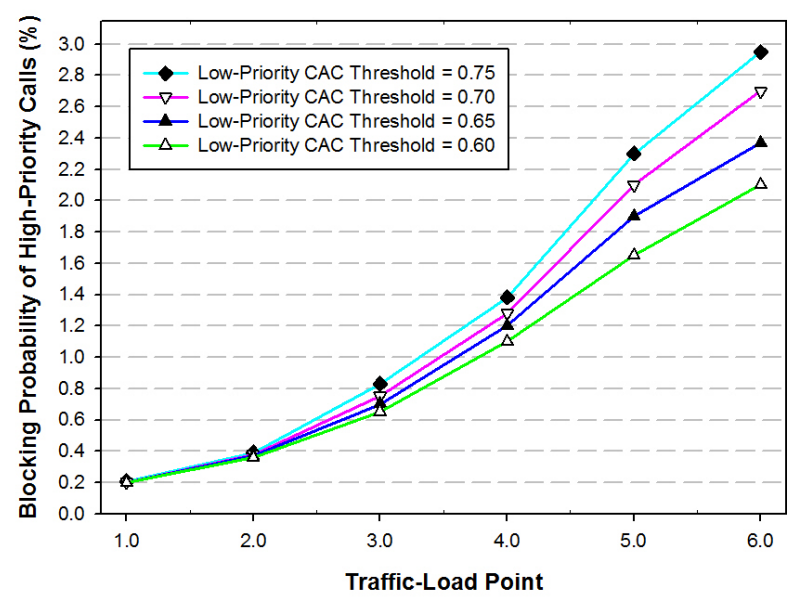

Fig. 8 Blocking probability of high priority calls vs CAC threshold of low priority calls (high traffic-load).

Table 2 Offered Traffic-Load $\left(a_{k, p}\right)$ in erl

\begin{tabular}{|c|c|c|}
\hline Traffic-Load & $1^{\text {st }}$ Service & $2^{\text {nd }}$ Service \\
\hline 1 & $a_{1,1}=0.02, a_{1,2}=0.1$ & $a_{2,1}=0.01, a_{2,2}=0.05$ \\
\hline 2 & $a_{1,1}=0.04, a_{1,2}=0.2$ & $a_{2,1}=0.02, a_{2,2}=0.10$ \\
\hline 3 & $a_{1,1}=0.06, a_{1,2}=0.3$ & $a_{2,1}=0.03, a_{2,2}=0.15$ \\
\hline 4 & $a_{1,1}=0.08, a_{1,2}=0.4$ & $a_{2,1}=0.04, a_{2,2}=0.20$ \\
\hline 5 & $a_{1,1}=0.10, a_{1,2}=0.5$ & $a_{2,1}=0.05, a_{2,2}=0.25$ \\
\hline 6 & $a_{1,1}=0.12, a_{1,2}=0.6$ & $a_{2,1}=0.06, a_{2,2}=0.30$ \\
\hline
\end{tabular}

priority traffic of 0.05 erl. We then increase the high-priority traffic up to 0.06 erl in steps of 0.01 erl, and the low-priority traffic up to $0.3 \mathrm{erl}$ in steps of $0.05 \mathrm{erl}$. The number of traffic sources for the first service is 120 . Out of them, 20 are the sources for high-priority calls and 100 are the sources for low-priority calls. The number of traffic sources for the second service is 60 , with 10 and 50 sources for high-priority and low-priority calls, respectively.

In Figs. 4 and 5, we present the analytical and simulation CBP for the first and the second service, respectively. The analytical results are very close to simulation results in all cases. This means that the accuracy of the proposed model is very good. We also observe that, for both services, the CBP of high priority calls are significantly lower than those of low priority calls. This has been achieved by selecting different $\mathrm{CAC}$ thresholds for high and low priority calls. In this example we used the following thresholds: $C L_{1, \max }=0.8$ and $C L_{2, \max }=0.7$.

As a second example, we study the impact of the CAC threshold of low-priority calls on the CBP of high-priority calls. We consider two different cases for the total offered traffic-load (low and high traffic) and increase the threshold of low-priority calls from 0.5 to 0.75 in steps of 0.05 .

- Low Traffic Case. The offered traffic-load for the first service is 0.48 erl. Out of this, 0.08 erl are for the highpriority calls and 0.4 erl are for the low-priority calls. Similarly, the corresponding numbers for the second service are: $0.24 \mathrm{erl}, 0.04 \mathrm{erl}$ and $0.2 \mathrm{erl}$.
- High Traffic Case. The offered traffic-load for the first service is 0.72 erl, with 0.12 erl and 0.6 erl for the high-priority calls and for the low-priority calls, respectively. The corresponding numbers for the second service are $0.36 \mathrm{erl}, 0.06 \mathrm{erl}$ and $0.3 \mathrm{erl}$.

The resultant CBP for high-priority calls are shown in Figs. 6 and 7 for low and high traffic, respectively. We also present in Fig. 8 the CBP of high-priority calls for 6 trafficload points of Table 2 and for 4 different CAC thresholds. For simplicity, only the analytical results are shown. We observe, that the CBP of high-priority calls can be significantly increased if the CAC threshold of low-priority calls is kept at high levels. This is especially true when the load is high.

\section{Conclusion}

We propose a novel teletraffic model for the call-level analysis of priority-based cellular CDMA networks. We consider multiple services with finite source population and explicitly incorporate into the model different call priority classes. The CDMA system is described with a continuous-time Markov chain, which leads to efficient recursive formula for the calculation of system state probabilities. To achieve that, we introduce a number of approximations whose impact is negligible as confirmed by simulation results. We also perform a study of the impact of low-priority CAC thresholds on the blocking probabilities of high-priority calls. Our results show that careful design of CAC policies is required to guarantee an appropriate QoS of high-priority users.

\section{References}

[1] P. Demestichas, et al., "5G on the horizon: Key challenges for the radio-access network," IEEE Vehicular Technology Magazine, vol. 8, no. 3, pp. 47-53, Sept. 2013.

[2] A. Tetsushi, Y. Kishiyama, Y. Kakura, and D. Imamura, "Radio interface technologies for cooperative transmission in 3GPP LTE-A," IEICE Trans. Commun., vol. 94, no. 12, pp. 3202-3210, Dec. 2011.

[3] Z. Zaidi, V. Friderikos, and M. A. Imran, "Future RAN architecture: SD-RAN through a general-purpose processing platform," IEEE Vehicular Technology Magazine, vol. 10, no. 1, pp. 52-60, March 2015.

[4] V. Vassilakis, I. Moscholios, A. Bontozoglou, and M. Logothetis, "Mobility-aware QoS assurance in software-defined radio access networks: An analytical study," Proc. IEEE Soft5G, London, UK, April 2015.

[5] Z. Wang, S. Fan, and Y. Rui, "CDMA-FMT: A novel multiple access scheme for 5G wireless communications," Proc. 19th DSP, Hong Kong, August 2014.

[6] S. Chen and J. Zhao, "The requirements, challenges, and technologies for $5 \mathrm{G}$ of terrestrial mobile telecommunication," IEEE Commun. Magazine, vol. 52, no. 5, pp.36-43, May 2014.

[7] M. Glabowski, A. Kaliszan, and M. Stasiak, "Modeling productform state-dependent systems with BPP traffic," Performance Evaluation, vol. 67, no. 3, pp. 174-197, March 2010.

[8] J. S. Kaufman, "Blocking in a shared resource environment," IEEE Trans. Commun., vol. 29, no. 10, pp. 1474-1481, Oct. 1981.

[9] J. W. Roberts, "A service system with heterogeneous user requirements," Performance of Data Communications Systems and Their Applications, North Holland, Amsterdam, pp. 423-431, Oct. 1981.

[10] I. Moscholios, M. Logothetis, and G. Kokkinakis, "Call-burst blocking of ON-OFF traffic sources with retrials under the complete shar- 
ing policy," Performance Evaluation, vol. 59, no. 4, pp. 279-312, March 2005.

[11] M. Glabowski, M. Stasiak, and P. Zwierzykowski, "Modelling of virtualcircuit switching nodes with multicast connections," European Trans. Telecommun., vol. 20, no. 2, pp. 123-137, March 2009.

[12] G. Fodor and M. Telek, "Bounding the blocking probabilities in multirate CDMA networks supporting elastic services," IEEE/ACM Trans. Netw., vol. 15, no. 4, pp. 944-956, Aug. 2007.

[13] V. Vassilakis and M. Logothetis, "The wireless Engset multi-rate loss model for the handoff traffic analysis in W-CDMA networks," Proc. IEEE PIMRC, Cannes, France, pp. 1-6, Sept. 2008.

[14] K. Kuppuswamy and D. C. Lee, "An analytic approach to efficiently computing call blocking probabilities for multiclass WDM networks," IEEE/ACM Trans. Netw., vol. 17, no. 2, pp. 658-670, April 2009

[15] J. Vardakas, I. Moscholios, M. Logothetis, and V. Stylianakis, "An analytical approach for dynamic wavelength allocation in WDMTDMA PONs servicing ON-OFF traffic," Journal of Optical Commun. and Networking, vol. 3, no. 4, pp. 347-358, March 2011.

[16] V. Vassilakis, I. Moscholios, and M. Logothetis, "Performance evaluation of priority-based CDMA systems in the presence of multirate Poisson traffic," Proc. IEICE ICTF, Manchester, UK, June 2015.

[17] D. Staehle and A. Mäder, "An analytic approximation of the uplink capacity in a UMTS network with heterogeneous traffic," Proc. ITC, Berlin, Germany, Sept. 2003.

[18] M. Stasiak, A. Wisniewski, P. Zwierzykowski, and M. Glabowski, "Blocking probability calculation for cellular systems with WCDMA radio interface servicing PCT1 and PCT2 multirate traffic,” IEICE Trans. Commun., vol. 92-B, no. 4, pp. 1156-1165, 2009.

[19] V. Vassilakis, G. Kallos, I. Moscholios, and M. Logothetis, "The wireless Engset multi-rate loss model for the call-level analysis of W-CDMA networks," Proc. PIMRC, Athens, Greece, Sept. 2007.

[20] I. Moscholios, G. Kallos, V. Vassilakis, and M. Logothetis, "Congestion probabilities in CDMA-based networks supporting batched Poisson input traffic," Wireless Personal Communications, vol. 79, no. 2, pp. 1163-1186, Nov. 2014.

[21] M. Glabowski, S. Hanczewski, and M. Stasiak, "Modelling of cellular networks with traffic overflow," Mathematical Problems in Engineering, pp. 1-15, Jan. 2015.

[22] M. Sobieraj, M. Stasiak, J. Weissenberg, and P. Zwierzykowski, "Analytical model of the single threshold mechanism with hysteresis for multi-service networks," IEICE Trans. Commun., vol. 95, no. 1, pp. 120-132, Jan. 2012.

[23] M. Rasti and E. Hossain, "Distributed priority-based power and admission control in cellular wireless networks," IEEE Trans. Wireless Commun., vol. 12, no. 9, pp. 4483-4495, Sept. 2013.

[24] S. Hanczewski, M. Stasiak, and P. Zwierzykowski, "Modelling of the access part of a multi-service mobile network with service priorities," EURASIP Journal on Wireless Commun. and Networking, no. 1, pp. 1-14, July 2015.

[25] H. Akimaru and K. Kawashima, "Teletraffic: theory and applications," Springer Publishing Company, 1993.

[26] V. Vassilakis, I. Moscholios, and M. Logothetis, "Call-level performance modelling of elastic and adaptive service-classes with finite population,” IEICE Trans. Commun., vol. 91, no. 1, pp. 151-163, Jan. 2008.

[27] S. Ataman, and A. Wautier, "Perfect power control in a multiservice CDMA cellular system,” Proc. IEEE PIMRC, Lisbon, Portugal, pp. 1222-1226, Sept. 2002.

[28] J. Zhang and T. Konstantopoulos, "Multiple-access interference processes are self-similar in multimedia CDMA cellular networks," IEEE Trans. Information Theory, vol. 51, no. 3, pp. 1024-1038, March 2005.

[29] T. Guo, N. Wang, and R. Tafazolli, "Local mobility management for networked femtocells based on X2 traffic forwarding," IEEE Trans. Veh. Tech., vol. 62, no. 1, pp. 326-340, Jan. 2013.

[30] Simscript III, http://www.simscript.com [January 2016].

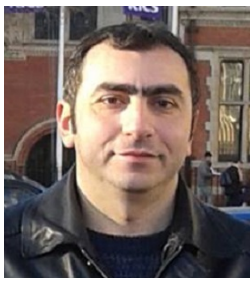

Vassilios G. Vassilakis received his Ph.D. degree in Electrical and Computer Engineering from the University of Patras, Greece in 2011. From 2011 to 2013 he was with the Network Convergence Laboratory (NCL), University of Essex, and conducting research on informationcentric networking and network security. From 2013 to 2015 he was with the Institute for Communication Systems (ICS), University of Surrey, and conducting research on fifth generation $(5 \mathrm{G})$ wireless networks. In 2015 he joined the Computer Laboratory, University of Cambridge. He's been involved in EU, UK, and industry funded R\&D projects related to the design of future mobile networks and new Internet architectures. His main research interests are in the areas of next-generation wireless and mobile networks, future Internet technologies, software-defined networks, and network security. He's published over 60 journal/conference papers. He's served as an Associate Editor in IEICE Transactions on Communications.

Ioannis D. Moscholios was born in Athens, Greece, in 1976. He received the Dipl.-Eng. degree in Electrical \& Computer Engineering from the University of Patras, Patras, Greece, in 1999 , the M.Sc. degree in Spacecraft Technology \& Satellite Communications from the University College London, UK, in 2000 and the Ph.D. degree in Electrical \& Computer Engineering from the University of Patras, in 2005. From 2005 to 2009 he was a Research Associate at the Wire Communications Laboratory, Dept. of Electrical \& Computer Engineering, University of Patras. From 2009 to 2013 he was a Lecturer in the Dept. of Telecommunications Science and Technology, University of Peloponnese, Tripolis, Greece. From 2013, he is an Assistant Professor in the Dept. of Informatics \& Telecommunications, University of Peloponnese, Tripolis, Greece. His research interests include teletraffic engineering, simulation and performance analysis of communication networks. He's published over 100 papers in international journals/ conferences. He's served as a Guest Editor in IET Networks and as an Associate Editor in IEICE Transactions on Communications. He is a member of the Technical Chamber of Greece (TEE).

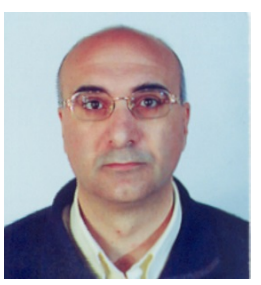

Michael D. Logothetis was born in Andros, Greece, in 1959. He received his Dipl.Eng. degree and Doctorate in Electrical Engineering, both from the University of Patras, Patras, Greece, in 1981 and 1990, respectively. From 1982 to 1990, he was a Teaching and Research Assistant at the Laboratory of Wire Communications, University of Patras, and participated in many national and EU research programmes, dealing with telecommunication networks and office automation. From 1991 to 1992 he was Research Associate in NTT's Telecommunication Networks Laboratories, Tokyo, Japan. Afterwards, he was a Lecturer in the Dept. of Electrical \& Computer Engineering of the University of Patras, and in 2009 elected (Full) Professor in the same Department. His research interests include teletraffic theory and engineering, traffic/network control, simulation and performance optimization of telecommunications networks. He's published over 180 conference/journal papers and have over 600 thirdpart citations. He's published a teletraffic book in Greek. He's organised the IEEE/IET CSNDSP 2006, and IEICE ICTF 2016. He's served as TPC member of several international conferences while he's chaired several technical sessions. He has become a Guest Editor in five journals: a) Mediterranean Journal of Electronics and Communications, b) Mediterranean Journal of Computers and Networks, c) IET Circuits, Devices \& Systems, d) IET Networks, and e) Ubiquitous Computing and Communication Journal. He is a member of the IARIA (Fellow), IEEE (Senior), IEICE, FITCE and the Technical Chamber of Greece (TEE). 